One is thus led to the conclusion that

$$
\lim _{a \rightarrow 0} 2 \cdot 2^{1 / 2} \int_{0}^{a}(F(a)-F(x))^{-1 / 2} d x=2 \pi b^{-1 / 2} .
$$

Hence we have the following theorem.

THEOREM 3. The period of vibration $T$ under restoring force $f(x)$, conditioned by hypotheses (A), (B), and (C), approaches the limit $2 \pi b^{-1 / 2}$ as the amplitude approaches zero.

Schenectady, N. Y.

\title{
A NOTE ON FERMAT'S LAST THEOREM
}

$$
\text { D. H. LEHMER* }
$$

In $1925 \mathrm{H}$. S. Vandiver $†$ proved the following theorem.

THEOREM 1. If

$$
x^{p}+y^{p}+z^{p}=0
$$

is satisfied by integers $x, y, z$, prime to the odd prime $p$, then the first factor of the class number of the field generated by $e^{2 \pi i / p}$ is divisible by $p^{8}$.

In the seventh of a series of articles on Fermat's last theorem, T. Morishima $\ddagger$ has given the following improvement upon Theorem 1.

Theorem 2. In Theorem 1 we may replace $p^{8}$ by $p^{12}$ provided $p$ does not divide $75571 \cdot 20579903$.

It is the purpose of this note to show that the proviso of Theorem 2 is unnecessary by showing that (1) is not satisfied by the prime factors of $75571 \cdot 20579903$. This is done by applying Wieferich's $\|$ criterion.

TheOREM 3. If (1) is satisfied by integers $x, y, z$, prime to $p$, then $2^{p-1} \equiv 1\left(\bmod p^{2}\right)$.

* National Research Fellow.

$\dagger$ Annals of Mathematics, (2), vol. 26, p. 232.

$\ddagger$ Proceedings of the Imperial Academy of Japan, vol. 8 (1932), pp. 63-66.

\| Journal für Mathematik, vol. 136 (1909), p. 203. 
In the first place

$$
p_{1}=75571 \text { and } p_{2}=20579903
$$

are prime numbers. To show that $p_{2}$ is a prime we observe that $2^{\left(p_{2}-1\right) / 563}=2^{36554} \equiv 18351241 \equiv r\left(\bmod p_{2}\right)$ and that $r-1$ and $p_{2}$ are relatively prime. By the congruence (2) below we have

$$
2^{p_{2}-1} \equiv 1 \quad\left(\bmod p_{2}\right) \text {. }
$$

Hence all factors of $p$ are of the form $563 x+1$. $^{*}$ There are no primes of this form less than the square root of $p_{2}$. Hence $p_{2}$ is a prime.

We find next that

$$
2^{p_{1}-1} \equiv 4481813727=1+59306 p_{1}\left(\bmod p_{1}^{2}\right)
$$

and

(2) $2^{p_{2}-1} \equiv 70637882819917=1+3432372 p_{2} \quad\left(\bmod p_{2}^{2}\right)$.

Hence, by Theorem 3 , equation (1) has no solutions $x, y, z$, prime to $p$ for $p=p_{1}$ or $p_{2}$. We have then the following lemma.

THEOREM 4. If $x^{p}+y^{p}+z^{p}=0$ has a solution for which $x y z$ and $p$ are coprime, then the first factor of the class number of the cyclotomic field $K\left(e^{2 \pi i / p}\right)$ is divisible by $p^{12}$.

StanFord University

* See this Bulletin, vol. 33 (1927), p. 331. Theorem 3. 